\title{
Multiplex Fluorescence Microscopy Platform for High-resolution ex Vivo and in Vitro imaging to Support Correlative Tissue Distribution and Biomarker Analysis
}

Niyanta Kumar and Mark Cancilla

Merck, West Point, Pennsylvania, United States

Drug delivery to the appropriate tissue and cellular compartment is often a key factor contributing to the success of macromolecular therapeutics such as proteins, peptides, oligonucleotides, and mRNA. The Pharmacokinetics, Pharmacodynamics, and Drug Metabolism group at Merck \& Co., Inc. is building a diverse tool kit to evaluate drug disposition for our therapeutic pipeline. Most pharmacokinetic studies currently depend on bulk sampling of plasma or tissue which does not capture the spatial heterogeneity in drug distribution at a high resolution. We are therefore developing several in vitro and ex vivo imaging methods to evaluate the distribution of therapeutic agents in cell culture systems and preclinical species. Advances in the range of fluorophores and fluorescent proteins, ease of labeling molecules with fluorophores, fluorescence microscopy resolution, and image analysis capabilities have made fluorescence-based imaging an attractive method to evaluate the distribution of macromolecular therapeutics.

We are currently applying fluorescence-based imaging using confocal microscopy to answer diverse questions for a wide spectrum of therapeutic modalities including mRNA vaccines, cell penetrating peptides, and antibodies among others. Our in vitro imaging studies aim to (i) understand mechanisms of cell uptake, (ii) quantify the efficiency of drug delivery to the cytosol and escape from the endosomal/lysosomal compartments, (iii) compare delivery efficiency across different nanoparticle carriers and formulations, (iv) understand the kinetics of drug distribution and trafficking within cells (v) and better define structure activity relationships and influence drug design. Ex vivo fluorescence-based imaging to examine drug, target, and biomarker distribution within tissues in preclinical species is being performed using immunofluorescence and fluorescence in situ hybridization. We are also evaluating tissue clearing techniques and light sheet microscopy for whole organ or tumor imaging at a high resolution. Here, we will share examples of how we are applying these varied ex vivo and in vitro fluorescence imaging approaches to support our therapeutic pipeline.

\section{References}

- Chung, K., Wallace, J., Kim, S. Y., Kalyanasundaram, S., Andalman, A. S., Davidson, T. J., Mirzabekov, J. J., Zalocusky, K. A., Mattis, J., Denisin, A. K., Pak, S., Bernstein, H., Ramakrishnan, C., Grosenick, L., Gradinaru, V. and Deisseroth, K. (2013) 'Structural and molecular interrogation of intact biological systems', Nature, 497(7449), pp. 332-7.

- Kumar, N. N., Pizzo, M. E., Nehra, G., Wilken-Resman, B., Boroumand, S. and Thorne, R. G. (2018) 'Passive Immunotherapies for Central Nervous System Disorders: Current Delivery Challenges and New Approaches', Bioconjug Chem, 29(12), pp. 3937-3966. 